\title{
SURVEY OF SOME RECENT STELLAR-EVOLUTION CALCULATIONS
}

Allen V. Sweigart

NASA Goddard Space Flight Center

\section{INTRODUCTION}

A basic objective of stellar-evolution theory is to provide detailed quantitative information on the evolutionary characteristics of stars with different compositions and masses. For the evolutionary phases from the zero-age main sequence (ZAMS) through core-helium burning the important physical processes are sufficiently well understood to justify a systematic investigation, and, indeed, extensive grids of evolutionary sequences have been constructed for these phases by many researchers. However, several difficulties are sometimes encountered when using the available calculations. For example, differences in numerical techniques and physical assumptions can give rise to systematic differences among the results of various investigations. Furthermore, the available calculations do not always explore the full ranges of the input parameters, and in some cases they neglect physical effects that are now believed to be important.

In an attempt to overcome some of these limitations, I have undertaken a series of computational projects covering the mainsequence, red giant and horizontal-branch phases. The immediate aim has been to produce a self-consistent set of evolutionary sequences for wide ranges in the composition and mass. The longterm aim involves the detailed application of these results to observational data on cluster HR diagrams and the integrated properties of galaxies. Other persons who have contributed significantly to various aspects of this work include P. Demarque, $J$. Mengel and P. Gross. 
TABLE I

MAIN-SEQUENCE-TURNOFF AND RED-GIANT SEQUENCES

\begin{tabular}{|l|l|l|l|}
\hline \hline$Y$ & $Z$ & $M_{\odot} M_{\odot}($ Turnoff) & $M_{\odot} M_{\odot}$ (Red Giant) \\
\hline 0.10 & 0.00001 & $0.70-4.40$ & 0.90 \\
0.20 & 0.00001 & $0.70-3.50$ & $0.70,0.90$ \\
0.30 & 0.00001 & $0.55-3.50$ & $0.70,0.90$ \\
0.40 & 0.00001 & $0.55-3.50$ & 0.70 \\
0.10 & 0.0001 & $0.70-4.40$ & 0.90 \\
0.20 & 0.0001 & $0.70-3.50$ & $0.70,0.90,1.40$ \\
0.30 & 0.0001 & $0.55-3.50$ & $0.70,0.90,1.40$ \\
0.40 & 0.0001 & $0.55-3.50$ & 0.70 \\
0.20 & 0.0004 & $0.70-3.50$ & 0.90 \\
0.30 & 0.0004 & $0.55-3.50$ & 0.70 \\
0.10 & 0.001 & $0.70-4.40$ & 0.90 \\
0.20 & 0.001 & $0.70-3.50$ & $0.70,0.90$ \\
0.30 & 0.001 & $0.55-3.50$ & $0.70,0.90$ \\
0.40 & 0.001 & $0.55-3.50$ & 0.70 \\
0.20 & 0.004 & $0.70-4.40$ & 0.90 \\
0.30 & 0.004 & $0.70-3.50$ & 0.70 \\
0.10 & 0.01 & $0.90-4.40$ & $0.90,1.10$ \\
0.20 & 0.01 & $0.70-4.40$ & $0.90,1.10,1.40$, \\
0.30 & 0.01 & $0.70-3.50$ & $0.70,0.90,1.10,1.75,2.20$ \\
0.40 & 0.01 & $0.55-3.50$ & $0.70,1.10$ \\
0.20 & 0.04 & $0.90-4.40$ & $0.90,1.75$ \\
0.30 & 0.04 & $0.70-4.40$ & $0.70,1.75$ \\
0.20 & 0.10 & $0.90-6.90$ & - \\
0.30 & 0.10 & $0.70-5.50$ & - \\
\hline
\end{tabular}

A listing of the computed sequences is provided in Tables I and II for all values of the helium abundance $Y$ and the heavyelement abundance $Z$ studied so far. The turnoff sequences extended from the ZAMS to the red giant branch except for a few sequences with high mass and low $Z$. The range in mass $M$ given in column 3 of Table I was covered by approximately 10 turnoff sequences having roughly equally spaced values of $\log M$. The red giant sequences listed in column 4 were continued to the onset of helium burning which in nearly all cases occurred under degenerate conditions. The quantity $\mathrm{M}_{\mathrm{c}}$ in Table II denotes the mass within the helium core at the zero-age horizontal-branch (ZAHB) phase.

The stellar-evolution program developed by Sweigart (1972, 1973) from the Princeton program (Schwarzschild and Härm 1965) was used for all computations to insure the self-consistency of the results. A more complete description of this program can be found in Sweigart and Gross (1974, 1978). Modifications were 
TABLE II

HORIZONTAL-BRANCH SEQUENCES

\begin{tabular}{|l|l|l|l|}
\hline \hline$Y$ & $Z$ & $M_{C} / M_{\odot}$ & \multicolumn{1}{|c|}{$M_{\odot} / M_{\odot}$} \\
\hline 0.10 & 0.0001 & 0.475 & $0.50(0.04) 0.90$ \\
0.20 & 0.0001 & 0.475 & $0.50(0.04) 0.90$ \\
0.30 & 0.0001 & 0.475 & $0.50(0.04) 0.90$ \\
0.40 & 0.0001 & 0.475 & $0.50(0.04) 0.98$ \\
0.20 & 0.0001 & 0.525 & $0.54(0.04) 0.90$ \\
0.30 & 0.0001 & 0.525 & $0.54(0.02) 0.58(0.04) 0.90$ \\
0.40 & 0.0001 & 0.525 & $0.54(0.04) 1.02$ \\
0.30 & 0.001 & 0.425 & $0.46(0.04) 0.90$ \\
0.40 & 0.001 & 0.425 & $0.46(0.04) 0.90$ \\
0.10 & 0.001 & 0.475 & $0.50(0.04) 0.90$ \\
0.20 & 0.001 & 0.475 & $0.50(0.04) 0.90$ \\
0.30 & 0.001 & 0.475 & $0.50(0.04) 1.02$ \\
0.40 & 0.001 & 0.475 & $0.54(0.04) 0.90$ \\
0.10 & 0.001 & 0.525 & $0.54(0.04) 0.90$ \\
0.20 & 0.001 & 0.525 & $0.54(0.04) 0.90$ \\
0.30 & 0.001 & 0.525 & $0.54(0.04) 1.06$ \\
0.40 & 0.001 & 0.525 & $0.58(0.04) 1.06$ \\
0.30 & 0.001 & 0.575 & $0.50(0.02) 0.58(0.04) 0.94$ \\
0.30 & 0.01 & 0.475 &
\end{tabular}

made to this program during the course of these projects in order to incorporate those additional physical effects of particular importance for each evolutionary phase. For example, relativistic degeneracy and semiconvection were included in the red giant and horizontal-branch sequences, respectively. It is well-known that the CNO abundance can significantly influence the characteristics of the main-sequence turnoff, and consequently the approach to equilibrium of the on cycle was followed during the turnoff calculations. The cNO abundance has been assumed here to scale with the $\mathrm{Fe}$ abundance, although there is observational evidence indicating that $\mathrm{CNO}$ and Fe may not be so tightly correlated.

The present results, consisting in total of over 100,000 stellar models, have a number of immediate astrophysical applications. From the fitting of theoretical isochrones with observed HR diagrams and from the relative red giant and horizontal-branch lifetimes one can in principle determine the ages and helium abundance of the globular clusters. Such information provides important constraints on cosmological models and has direct implications for the dynamical and chemical evolution of the Galaxy. The number distribution of stars along the giant branch can be compared with theoretical luminosity functions in an attempt to understand further the observed irregularities (Sandage et al. 
1968; Sweigart 1978). The present red giant models can serve as the starting point for subsequent studies of the helium-core flash (Mengel 1978) and of the unusual C, N and s-process abundances found in some globular-cluster stars (Mengel and Sweigart 1978). Furthermore it will be possible to consider again in more detail some of the intriguing problems associated with the morphology of the horizontal branch.

In the following sections I will survey the various evolutionary calculations. For conciseness the discussion must, however, be limited to a few representative cases. A more thorough description of the available data is contained in the cited references.

\section{TURNOFF SEQUENCES}

The turnoff tracks for $Z=0.01$ and $Y=0.20$ and 0.30 are shown, respectively, in Figs. 1 and 2 (Mengel et al. 1978). In the higher mass sequences central hydrogen exhaustion occurs throughout a rather large region at the end of the main-sequence phase. This leads to an abrupt contraction of the star and hence to a blueward fluctuation in the track. Observationally this fluctuation would appear as a gap in a cluster HR diagram. Although not plotted in Figs. 1 and 2, a few intermediate mass tracks have been constructed in order to define the development of the gap more precisely. As expected, an increase in $Y$ shifts

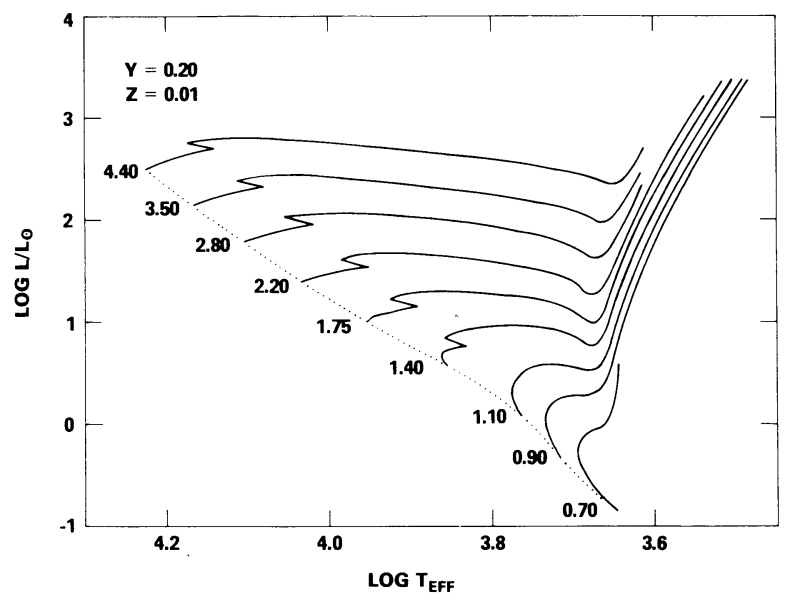

Fig. 1. Main-sequence-turnoff and red-giant tracks for $(Y, Z)=$ $(0.20,0.01)$. The location of the ZAMS is indicated by the dotted line. Each track is labeled by the mass in solar units. The tracks for $0.90 \leq \mathrm{M} \leq 2.20 \mathrm{M}_{\odot}$ have been evolved to the onset of helium burning. 


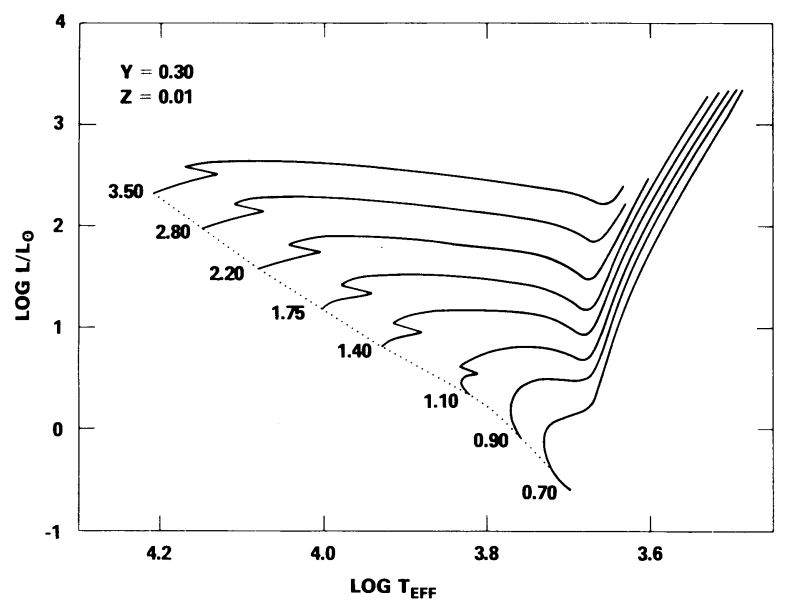

Fig. 2. Main-sequence-turnoff and red-giant tracks for $(Y, Z)=$ $(0.30,0.01)$. The location of the ZAMS is indicated by the dotted line. Each track is labeled by the mass in solar units. The tracks for $0.70 \leq M \leq 2.20 M_{\odot}$ have been evolved to the onset of helium burning.

a given track to larger values of log $\mathrm{T}_{\text {eff }}$ and Log $\mathrm{L}$.

The dependence of the turnoff tracks on $\mathrm{Z}$ is illustrated in Fig. 3. Simoda and Iben (1968) have demonstrated that an increase in $Z$ will reduce $\log T_{\text {eff }}$ and $\log L$ at the turnoff point. Such an effect is evident in Fig. 3 and is due primarily to the effects

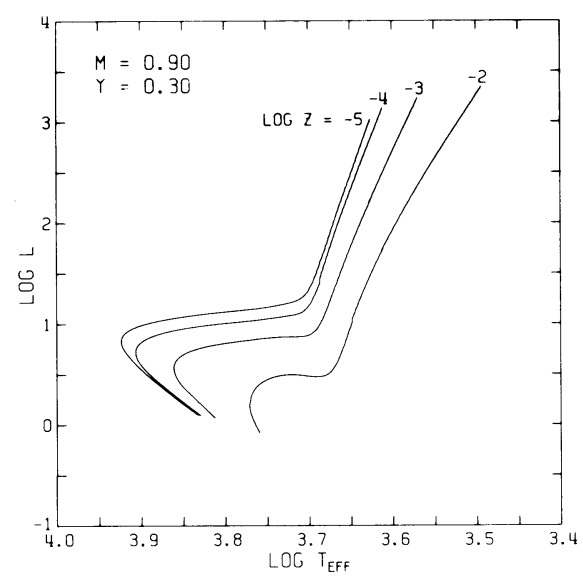

Fig. 3. Main-sequence-turnoff and red-giant tracks for $(M, Y)=$ $(0.90,0.30)$. Each track is labeled by the value of log $\mathrm{Z}$. 


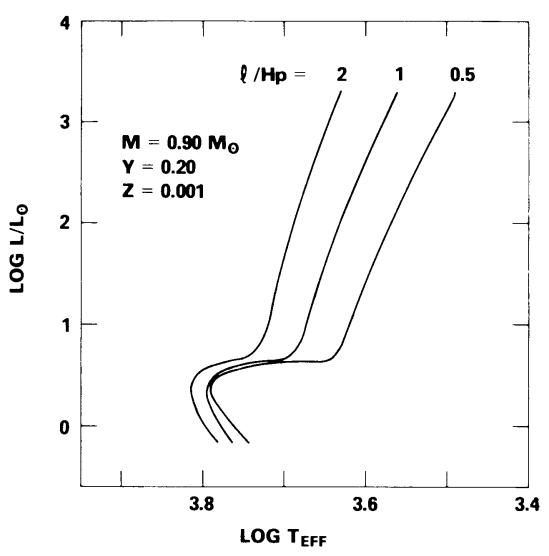

Fig. 4. Main-sequence-turnoff and red-giant tracks for (M, Y, Z) $=(0.90,0.20,0.001)$. Each track is labeled by the value of the mixing-length ratio $\alpha=\ell / H_{p}$.

of the CNO elements on the hydrogen-burning rate and the opacity (Renzini 1977). Any variations in the CNO abundance relative to the $\mathrm{Fe}$ abundance could therefore significantly influence the turnoff characteristics.

Since the lower mass sequences have a convective envelope, their tracks are affected by the value adopted for the ratio $\alpha$ of the mixing length $\ell$ to the pressure-scale height $\mathrm{H}_{\mathrm{p}}$ (Sweigart 1978). All of the present sequences use $\alpha=1$ except for the 2 cases plotted in Fig. 4. In this figure log Teff at the ZAMS and along the red-giant branch is approximately proportional to log $\alpha$. Not surprisingly, $\log \mathrm{L}$ is rather insensitive to the value of $\alpha$. Around the turnoff point the dependence of $\log \mathrm{T}_{\text {eff }}$ on $\log \alpha$ is quite nonlinear, since the envelope convection has almost disappeared in the $\alpha=0.5$ and 1 cases while it is still present in the $\alpha=2$ case. Sequences with a deeper convective envelope would be expected to show a smoother variation of log Teff at turnoff with $\log \alpha$. The caution which must be exercised when ages are obtained by fitting isochrones to cluster HR diagrams is indicated in Fig. 4. If the fit is based essentially on the turnoff color and if the correct $\alpha$ has not been used, the derived ages can be rather uncertain, a point emphasized by Iben and Rood (1970).

\section{RED-GIANT SEQUENCES}

Some of the red-giant tracks have already been displayed in Figs. I to 4. From these tracks and the others listed in Table I the following conclusions can be drawn (Rood 1972; Sweigart and 


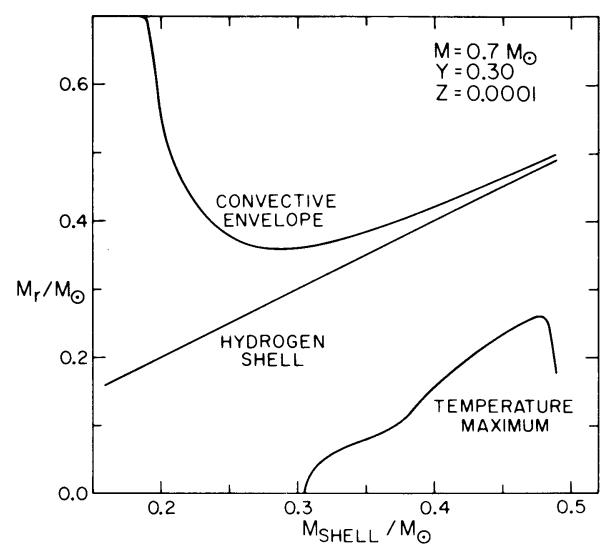

Fig. 5. Variations in the locations of the convective envelope, the hydrogen shell and the core-temperature maximum along a redgiant sequence for $(\mathrm{M}, \mathrm{Y}, \mathrm{Z})=(0.70,0.30,0.0001)$. The evolution between the end of the main-sequence phase and the onset of the helium-core flash is shown.

Gross 1978; Sweigart 1978). First, there is a blueward shift of the red giant branch with increasing $Y, M$ or $\alpha$ or decreasing $Z$. Second, an increase in $Y$ or a decrease in $Z$ lowers the surface luminosity at the tip of the red-giant branch and reduces the maximum stellar mass at which the helium-core flash still takes place.

How the edge of the convective envelope and the point of maximum temperature within the degenerate helium core vary along a typical red giant sequence is illustrated in Fig. 5. The abscissa gives the location of the center of the hydrogen shell, defined as the point where the hydrogen abundance is one half of its envelope value. The location of the hydrogen shell is thus represented by a straight line. The temperature inversion produced by neutrino emission becomes progressively more extensive as the mass within the core grows. Shortly before the helium-core flash the temperature maximum suddenly shifts inward. The flash point can be quite off center, especially for small values of $Y$ and $M$.

of particular interest is the behavior of the convective envelope. Initially the convective envelope moves inward in mass until deepest penetration is reached on the lower subgiant branch. At that time the convective envelope extends into the region that was partially depleted of hydrogen during the main-sequence phase. A discontinuity in the hydrogen abundance is therefore created at the point of deepest penetration. The convective envelope then retreats outward due to the approach of the hydrogen shell. When 


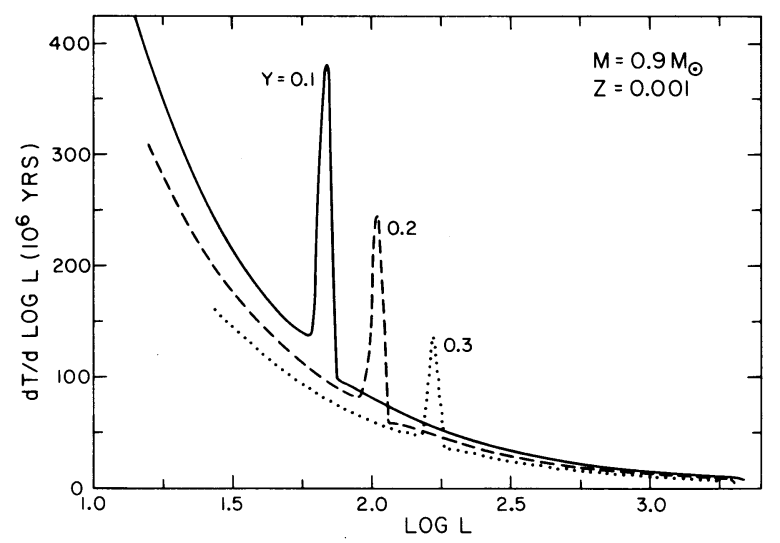

Fig. 6. Theoretical red-giant luminosity functions for $(M, Z)=$ $(0.90,0.001)$ and $Y=0.10,0.20$ and 0.30 . The ordinate gives the time $T$ spent per unit interval in $\log \mathrm{L}$ in units of $10^{6} \mathrm{yrs}$.

the hydrogen shell later encounters this discontinuity, a readjustment of the interior structure takes place, leading to a hesitation in the rate of evolution up the giant branch and often to a temporary drop in the surface luminosity (Rood 1972). The result can be a rather pronounced peak in the luminosity function as is demonstrated by Figs. 6 and 7 (Sweigart 1978). In general the prominence of this peak is increased and the luminosity at which it occurs is lowered by smaller values of $Y$ and larger values of $Z$. Such composition changes tend to produce a deeper

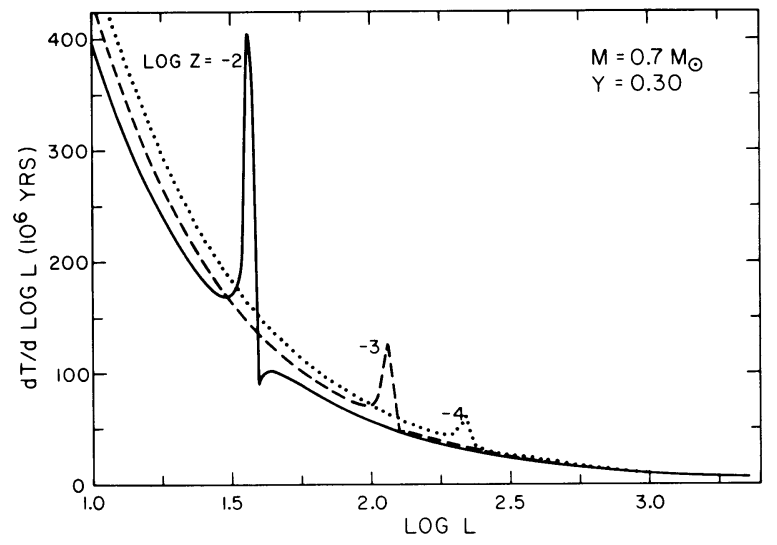

Fig. 7. Theoretical red-giant luminosity functions for ( $M, Y)=$ $(0.70,0.30)$ and $\log Z=-2,-3$ and -4 . The ordinate gives the time $\mathrm{T}$ spent per unit interval in $\log \mathrm{L}$ in units of $10^{6} \mathrm{yrs}$. 
penetration of the convective envelope on the subgiant branch and thus a more pronounced hydrogen discontinuity.

A major reason for constructing red-giant sequences is to determine the core mass $M_{C}$ at the time of the helium-core flash. The precise value of $M_{c}$ strongly affects the morphology of the horizontal-branch tracks as well as the horizontal-branch lifetimes. The present calculations give

$$
\begin{aligned}
\mathrm{M}_{\mathrm{C}} / \mathrm{M}_{\odot}= & 0.474-0.24(\mathrm{Y}-0.30)-0.007(\log \mathrm{Z}+3) \\
& -0.038(\mathrm{M}-0.80)
\end{aligned}
$$

in the neighborhood of the case $(\mathrm{M}, \mathrm{Y}, \mathrm{Z})=(0.80,0.30,0.001)$. Eqn. (1) assumes the neutrino emission rates of Beaudet et al. (1967). If neutrino emission is omitted, $M_{C}$ decreases by $0.03 M_{\odot}$, while the additional neutrino emission due to neutral current interactions raises $\mathrm{M}_{\mathrm{C}}$ by $0.007 \mathrm{M}_{\odot}$ (Sweigart and Gross 1978).

\section{HORIZONTAL-BRANCH SEQUENCES}

The interior structure and observable properties of horizontal-branch models are substantially altered by convective overshooting and semiconvection within the helium core (Schwarzschild 1970). During the initial horizontal-branch evolution the convective core propagates outward in mass because of convective overshooting. This growth in the convective core is driven fundamentally by the increase in the opacity and hence the radiative gradient caused by the gradual depletion of the central helium abundance (Castellani et al. 197la, b). Tentative estimates for the rate of propagation suggest that convective neutrality will, to a good approximation, be maintained at the convective-core edge (Renzini 1977). When the central helium abundance falls below about 0.7 , a semiconvective zone develops in the layers surrounding the convective core. The subsequent changes in the composition distribution are shown in Fig. 1 of Sweigart and Gross (1974). The effects of semiconvection on the evolution have been examined in a series of papers (Demarque and Mengel 1972; Sweigart and Demarque 1972; Sweigart and Gross 1974, 1976). The results show that semiconvection can almost double the horizontal-branch lifetime and track length when compared with calculations in which neither convective overshooting nor semiconvection is permitted.

Some typical horizontal-branch tracks computed with semiconvection (Sweigart and Gross 1976) are presented in Fig. 8. These tracks extend from the ZAHB to the initial helium-shell burning stage and are similar to those obtained by Gingold (1976). The 0.50 Mo track evolves vertically in the HR diagram and would not be expected to return to the asymptotic branch. The $0.54 \mathrm{M}_{\odot}$ track 


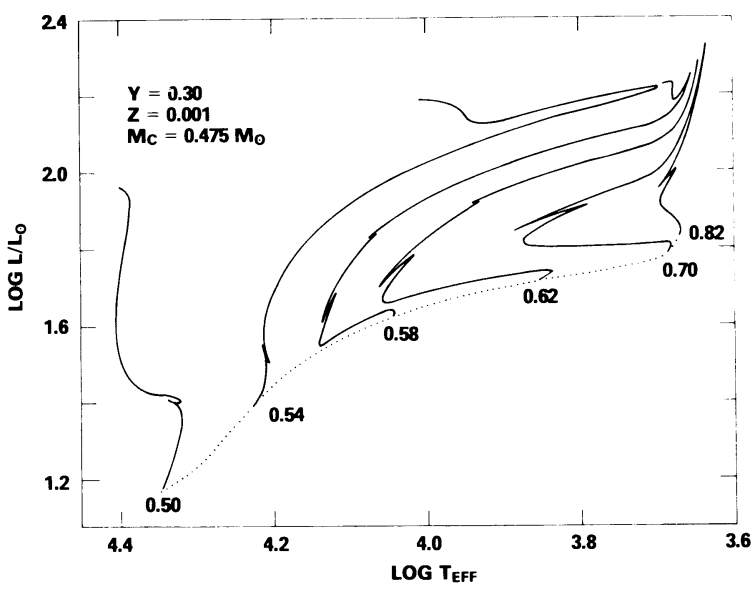

Fig. 8. Horizontal-branch and post-horizontal-branch tracks for $\left(Y, Z, M_{C}\right)=(0.30,0.001,0.475)$. The location of the ZAHB is indicated by the dotted line. Each track is labeled by the mass in solar units.

makes an excursion into the region of the BL Her stars in a manner similar to the $0.51 \mathrm{M}_{\odot}$ track of Sweigart et al. (1974). At higher masses the tracks all evolve back to the asymptotic branch. The spike visible at the end of the main horizontal-branch phase of each track is due to a rapid growth in the extent of the convective core and appears to be caused by an instability in the composition distribution within the core (Sweigart and Demarque 1973). The same effect has been reported by Gingold (1976) and Taam et al. (1976).

The author wishes to express his appreciation to G. Lasher, whose helpful contributions made much of this research possible, and to S. Sofia for constructive comments on the manuscript. The support of grant AST72-04418 A04 from the National Science Foundation is gratefully acknowledged.

\section{REFERENCES}

Beaudet, G., Petrosian, V. and Salpeter, E.E. (1967). Astrophys. J. 150,979 .

Castellani, V., Giannone, P. and Renzini, A. (1971a). Astrophys. Space Sci. 10, 340.

Castellani, V., Giannone, P. and Renzini, A. (1971b). Astrophys. Space Sci. 10, 355.

Demarque, P. and Mengel, J.G. (1972). Astrophys. J. 171, 583. Gingold, R.A. (1976). Astrophys. J. 204, 116. 
Iben, I., Jr. and Rood, R.T. (1970). Astrophys. J. 159, 605. Mengel, J.G. (1978). in preparation.

Mengel, J.G. and Sweigart, A.V. (1978). in preparation.

Menge 1, J.G., Sweigart, A.V., Demarque, P. and Gross, P.G. (1978). in preparation.

Renzini, A. (1977). Lectures given at the 7th Advanced Course on Advanced Stages in Stellar Evolution, Saas-Fee, Switzerland. Rood, R.T. (1972). Astrophys. J. 177, 681.

Sandage, A., Katem, B. and Kristian, J. (1968). Astrophys. J. Letters 153, L129.

Schwarzschild, M. (1970). Quart. J. Roy. Astron. Soc. 11, 12. Schwarzschild, M. and Härm, R. (1965). Astrophys. J. 1 42, 855. Simoda, M. and Iben, I., Jr. (1968). Astrophys. J. 152, 509. Sweigart, A.V. (1972). Bull. Am. Astron. Soc. 4, 203. Sweigart, A.V. (1973). Astron. Astrophys. 24, 459. Sweigart, A.V. (1978). in preparation.

Sweigart, A.V. and Demarque, P. (1972). Astron. Astrophys. 20, 445. Sweigart, A.V. and Demarque, P. (1973). In IAU Colloquium No. 21, Variable Stars in Globular Clusters and in Related Systems, J.D. Fernie, ed., D. Reidel, Dordrecht, p. 221.

Sweigart, A.V. and Gross, P.G. (1974). Astrophys. J. 190, 101.

Sweigart, A.V. and Gross, P.G. (1976). Astrophys. J. Suppl. 32, 367. Sweigart, A.V. and Gross, P.G. (1978). Astrophys. J. Suppl. in press. Sweigart, A.V., Mengel, J.G. and Demarque, P. (1974). Astron. Astrophys. 30,13 .

Taam, R.E., Kraft, R.P. and Suntzeff, N. (1976). Astrophys. J. 207, 201.

DISCUSSION

COX: Can you tell me what your best $\mathrm{Y}$ value is for the RR Lyrae stars?

SWEIGART: The value of $\mathrm{Y}$ can be estimated from the ratio $\mathrm{R}$ of the number of horizontal-branch stars to the number of red giant stars, since the corresponding ratio of theoretical lifetimes is a sensitive function of $Y$. The original number counts gave a value of 1 for $R$, implying a $Y$ value of about 0.3 . This $Y$ value was determined from a theoretical calibration in which the effects of semiconvection were neglected. It is now well-known that semiconvection can substantially increase the horizontal-branch lifetime. The resulting change in the calibration between $R$ and $Y$ reduces the $Y$ value for $R=1$ to 0.12 . Renzini (1977) has recently re-examined the number counts in more detail and has found that a significant number of faint horizontal-branch stars were omitted in the original counts. According to Renzini (1977), the average value of $R$ for a number of globular clusters is about 1.6. For this $R$ value one obtains $Y \sim 0.22$, when the calibration including semiconvection is used. This value for the helium abundance represents the best estimate currently available from the $R$ method. 
COX: You know, of course, that pulsation theory gives a value of about 0.3. Do you have any comment?

SWEIGART: All I can say is that the value based upon the number counts is about $0.20-0.25$. I do not think that 0.3 is possible. However, a detailed calibration of the $\mathrm{R}$ method using the latest horizontal-branch and red giant sequences has not yet been made, and thus the value I have given is a preliminary one.

ROOD: In reply to Cox's suggestion that RR Lyrae theory requires Y 0.28 to 0.30 , I think most of the indications, at least from the linear theory, are that Y 0.20 to 0.25 . Iben and Tuggle's blue edge calculations are consistent with this.

CASTELLANI: What is the dependence of "RG clumps" on the assumed value of the mixing length?

SWEIGART: The peak in the red-giant luminosity function does depend somewhat on the assumed value for the ratio, $\alpha$, of the mixing length to the pressure-scale height. An increase in $\alpha$ reduces the size of the peak and raises the Iuminosity $L$ at which it occurs. Although detailed luminosity functions have not yet been computed for $\alpha \neq 1$, this dependence appears to be mild. For example, in the case $(\mathrm{M}, \mathrm{Y}, \mathrm{Z})=(0.90,0.20,0.001)$, a change in $\alpha$ from 1 to 2 increases log $L$ at the peak by only 0.02 .

CASTELLANI: I have one more comment on the problem of the $R R$ Lyrae stars raised by $\mathrm{Dr}$. Cox. I know that in decreasing the helium content the width of the instability strip is expected to decrease. Nevertheless I wish to emphasize that, if one assumes a constant width of the strip (i.e. the observed one) and looks at the fitting of the observed period-frequency histograms then available pulsational theories, when connected with evolutionary prescriptions, clearly indicate a helium content on the order of $0.23-0.25$. On the contrary, no agreement can be found with the assumption that $\mathrm{Y}=0.30$ for the large majority of galactic globular clusters.

FLOWER: I have one comment to make concerning the proper choice of the value of the mixing length. The evolutionary tracks (Mengel, et. al., 1978) used to construct isochrones were computed using a value of 1.0 for the mixing length. Since, for the present generation of evolutionary codes, a value closer to 1.5 or 1.6 seems to be necessary to (1) reproduce the surface temperature of the present day Sun; (2) reproduce the location of the red giant branch on the HR diagram for old galactic clusters; and (3) reproduce the location of red giants on the HR diagram in young populous clusters in the IMC, the isochrones must be corrected to reflect these "calibrations" of the mixing length.

SWEIGART: I agree that a value closer to 1.5 gives a better fit with observations. It is particularly important to be aware of the dependence of the sequences on the mixing length when using the present results. 